\title{
Kritischer Forschungsabriss zum Terminus „Migrationsliteratur“
}

\begin{abstract}
Im Beitrag werden terminologische Fragen in Bezug auf den Begriff „Migrationsliteratur“ aufgegriffen, wobei vornehmlich die Substitution des genannten Begriffs durch neuere Termini gesichtet wird. Es wird für einen breit verstandenen Migrationsbegriff plädiert, wobei „Migration“ als Zirkulation zwischen Räumen verstanden wird, „Migrationsliteratur“ ein äußerst hybrides ästhetisches Phänomen zum Ausdruck bringt, und die Berücksichtigung der Biographie vielmehr im Hintergrund der Entstehungs- und Existenzbedingungen dieser Literatur zu sichten ist. Schließlich sind komparatistische Studien im Sinne von „Europäischen Migrationsliteraturen“ vonnöten, da dadurch verstärkt die Kontexte der Migration in Europa miteinander in Beziehung gesetzt werden können. Dies wiederum würde die Erforschung eines gemeinsamen interkulturellen Gedächtnisses in Europa unterstützen.
\end{abstract}

Keywords: Terminologie; Migrationsliteratur; Migration; Interkulturalität; Komparatistik

Es ist längst bewiesen, dass die Literaturwissenschaft nicht neutral sein kann, Vorstellungen und Einsichten lenkt, reale Auswirkungen auf Verfahrensweisen forciert. Im folgenden Beitrag wird auf der Basis dieser Einsicht eine kritische Diskussion zum Terminus „Migrationsliteratur“ vorgenommen, wobei der Fokus den Argumenten für die Substitution von „Migrationsliteratur“ durch andere Termini, wie z.B. „Interkulturelle Literatur“ oder „Literatur ohne festen Wohnsitz“, gilt. Meine These ist dabei, dass neuere Definitionen dem Terminus „Migrationsliteratur“ eine ausschließlich defizitäre Semantik unterstellen und dann, auf der Basis der Ablehnung dieses Terminus, die eigenen Vorschläge entgegengehalten. Dabei wird selten, dies sei hier vorweggenommen, das Verständnis der „Migration“ umrissen, was jedoch eine unabdingbare Mühe im Rahmen terminologischer Abgrenzungen ist, zumal es sich um ein literarisches Phänomen handelt, das vielfältig auf den Diskurs der Migration reagiert. Insofern wird im folgenden Beitrag für den strategischen Einsatz von „Migrationsliteratur“ plädiert, damit sowohl der historische Entstehungskontext als auch gleichzeitig die positiven Aspekte, ergo die ästhetisch-interkulturelle Konstitution dieser Literatur zum Ausdruck gebracht werden, wohlwissend, dass die Literaturwissenschaft durchaus Begrifflichkeiten konnotiert. 


\section{Zum Vermächtnis der „Gastarbeiterliteratur“}

Zunächst als „Gastarbeiterliteratur"1 bezeichnet, bestimmte in den achtziger Jahren Weinrich diese Literatur als deutsche „Literatur der Betroffenheit“ (Weinrich 1984, 22), was wiederum Ackermann mit dem Konzept der „Ausländerliteratur“ zu relativieren suchte. „Ausländerliteratur“ bezeichne einen Teil der deutschen Literatur, dürfe aber nicht von ihr vereinnahmt werden (vgl. Ackermann 1993, 55). Die Bereicherung, die Ackermann behauptet, wird jedoch von Adelson als ethnozentrisch verstanden, da durch die ausländischen Zusätze die Vorstellung des deutschen Kerns der Gegenwartsliteratur - was ist wirklich deutsch nicht hinterfragt werde (vgl. Adelson 1991, 68).

Bleiben wir aber kurz beim Begriff der „Gastarbeiterliteratur“. Im Interview von Julia Abel mit Feridun Zaimoglu mit dem Titel „Migrationsliteratur ist ein toter Kadaver“ der Zeitschrift Text+Kritik von 2006 äußert Zaimoglu, dass die Migrationsliteratur keine Rolle mehr spiele, obgleich er nicht wisse, ob man mit Migrationsliteratur die Gastarbeiterliteratur der ersten Stunden meine „und in der Verlängerung dann diese Weinerlichkeit, das kultivierte Fremdsein [...]“ (Zaimoglou 2006, 162). Hier sieht man ganz deutlich, dass die Migrationsliteratur stark in die Nähe der Gastarbeiterliteratur rückt und diese wiederum ausschließlich mit den literaturwissenschaftlich pejorativen Attributen des Betroffenheitsdiskurses versehen wird. Wie aber Abel in ihrem eigenen Artikel im genannten Band feststellt, gelte zumindest in den ersten Anthologien des PoLiKunst-Vereins (Polynationaler Literatur- und Kunstverein $)^{2}$ ein spielerischer Umgang mit dem Schubladendenken in Bezug auf Gastarbeiterliteratur, denn Begriffe wie „Gastarbeiter“ und „Gastarbeiterdeutsch" würden durchaus ironisch eingesetzt. Wichtig ist dabei ihre Feststellung, dass Topoi der damaligen Diskussion von der Bereicherung der deutschen Literatur durchaus bis heute die Debatten um „Migrationsliteratur“ bestimmen (vgl. Abel 2006, 237). Es ist deutlich, dass Zaimoglu den Begriff der „Gastarbeiterliteratur“ im semantischen Licht des „Gastarbeiters“ sieht, was linguistisch betrachtet die soziale, kulturelle und sprachliche Marginalität konnotiert, der Begriff stelle „fremdethnische Zugehörigkeit immer in Relation zu sozialen, sprachlichen und kulturellen Defizitkategorien [her]“ (Hinnenkamp 1990, 277).

Die Identifikation jedoch der Gastarbeiter- mit der Migrationsliteratur ist ebenso an literaturwissenschaftlichen Arbeiten der achtziger Jahre zu konstatieren. Zu erinnern ist an die Forschungsarbeiten von Heimke Schierloh (1984) Das

1 Ausführliche Beschreibungen zu älteren Begriffszuweisungen vgl. Esselborn 1995, 411-427; Blioumi 2001, 112-116.

2 Weitere Informationen dazu vgl. Weigel 1992, 210. 
alles für ein Stück Brot. Migrantenliteratur als Objektivierung des „Gastarbeiterdaseins“ und Horst Hamm (1988) Fremdgegangen - freigeschrieben. Eine Einführung in die deutschsprachige Gastarbeiterliteratur. Nicht zu verkennen ist dabei die Tatsache, dass die damalige „Gastarbeiterliteratur“ in einem sozialpädagogischen Kontext gesichtet wurde. Schließlich hat Amodeo in ihrer Pionierarbeit bewiesen, dass selbst die Literaturwissenschaft diese Literatur entfiktionalisiert, psychologisiert, trivialisiert, stereotypisiert, exotisiert hat (vgl. Amodeo 1996). Vor diesem Hintergrund möchte ich nun die neueren Definitionen vorstellen.

\section{Migrantenliteratur und Migrationsliteratur}

Nach Dörr (2009, 59f.) ist aufgrund des/der cultural turn/s die Literatur von ehemaligen Gastarbeitern und ihren Nachkommen, die nun „Menschen mit Migrationshintergrund“ heißen, in ständigem Wechsel mit ihren Leitkonzepten, wobei es für ihn nicht von der Hand zu weisen ist, dass es sich vielmehr um rhetorische Kosmetika handle. Weniger neutral fallen die Ausführungen von Lübcke aus, die nicht den Begriff „Migrationsliteratur“, sondern ausschließlich den Begriff „Migrantenliteratur“ fokussiert, worunter die „direkte biographische Verknüpfung von Literatur und Migration“ gemeint ist. Letztere Bezeichnung sei jedoch eine Etikettierung, die eine Reihe von Fragen aufwerfe, wie z. B., ob es sich um Literatur handle, die Migration behandelt, ob die Schreiber Migrantinnen und Migranten sind - oder gar die Leser (!) -, welche räumlichen Bewegungen sie für diese Kategorisierungen vollziehen müssen. Diese in plakativem Modus formulierten Fragen finden laut Lübcke ihre Berechtigung nur, wenn der Begriff Migrantenliteratur ,als politische Kategorie strategisch eingesetzt wird“. (Lübcke 2009, 78) Zweifelsohne entspringt die begriffliche Schwierigkeit dieser Literatur der Tatsache, dass sie weder anhand deutlicher außerliterarischer oder biographischer Kriterien noch anhand ästhetischer, formaler oder thematischer Aspekte charakterisiert werden kann, was dazu führt, dass der herkömmliche begriffliche Kontext der germanistischen Literaturwissenschaft infrage gestellt wird (vgl. Schmitz 2009, 9). Es ist jedoch fraglich, ob auf den Begriff Migrant oder dessen Ableitungen ganz zu verzichten ist, obwohl sich diese Literatur auf den Diskurs der Migration bezieht, und statt dessen der Begriff „transnationale Literatur“ vorzuziehen ist, wie Lübcke vorschlägt, da vermeintlich dadurch „Reproduktionen homogener Zuschreibungen“ (Lübcke 2009, 78) weitgehend eingeschränkt werden.

Positiv wird der Begriff in der Studie von Schenk konnotiert, zumal der Sammelband den Titel Migrationsliteratur trägt. Im Vorwort wird für einen offen gehaltenen Begriff von „Migrationsliteratur“ plädiert, der unterschiedliche Facet- 
ten und Ausprägungen meint (vgl. Schenk et al. 2004, X). Es ist offenkundig, dass darunter eher innerliterarische Aspekte gesichtet werden, da als „Schreibweisen der Migrationsliteratur“ (Schenk et al. 2004, IX) diejenigen verstanden werden, die der kulturellen Vielfalt der deutschsprachigen Literatur Rechnung tragen. Der Begriff der „Migrationsliteratur“ wird bei allen Beiträgen des Sammelbands stets respektiert, gelegentlich jedoch gibt es Abgrenzungen konkret zum Begriff der „Migrant(en)literatur“. Es werden z. B. bei Kliems „Gastarbeiterliteratur“, „Ausländerliteratur“ und „Migrantenliteratur“ als „dilemmatische Hilfskonstrukte“ bezeichnet, die auf den politisch-sozialen Sonderstatus der Autoren mit Migrationshintergrund rekurrieren (Kliems 2004, 299).

Im Zuge der Kontrastierung zwischen „Migrantenliteratur“ und „Migrationsliteratur“ scheint sich letzterer durchgesetzt zu haben, da er unter anderem, so wie ihn Heidi Rösch $(1992,32)$ geprägt hat, Neutralität zum Ausdruck bringe. Trotzdem gibt es nicht die eine grundlegende Definition von „Migrationsliteratur“, auch wenn Weitin argumentiert, dass der Begriff am häufigsten in der Art gebraucht wird, wie ihn „Blioumi konsequent in ihren Arbeiten verwendet hat“ (Weitin 2012, 219). Dieser Begriff wiederum wird heuristisch verstanden und fasst die Dimensionen Thematik, Herkunft und Sprache als relationale Größen auf, die je nach Kombination im jeweiligen Text beachtet werden. Ausgangspunkt ist dabei ein breit verstandener dekonstruktiver Migrationsbegriff, demzufolge „Migration“ als Zirkulation zwischen Räumen verstanden wird und „Migrationsliteratur“ ein äußerst hybrides ästhetisches Phänomen zum Ausdruck bringt (vgl. Blioumi 2006, 19). Die Biographie des Autors sei dabei nicht als Ordnungskategorie zu verstehen, die die Zugehörigkeit zu einer Nationalliteratur bestimmt. Die Berücksichtigung der Biographie ist vielmehr im Hintergrund der Enstehungs- und Existenzbedingungen dieser Literatur zu sehen, denn durch die Sichtung der Biographie können unterschiedliche literarische Traditionen, sprachliche Dialogizität und kulturelle Hybridität festgestellt werden. Migrationsliteratur konstituiert sich demzufolge aus dem möglichen Zusammenspiel biographischer, thematischer, sprachlicher, ästhetischer und interkultureller Merkmale (vgl. Blioumi 2000, 599), wobei der breite Begriff unverkennbar eine breite Beschreibungsspanne zur Folge hat. Interessanterweise grenzt Weitin den Begriff „Migrationsliteratur“ von „Migrantenliteratur“ ab und meint, dass letzterer an exponierten Stellen doch noch gelegentlich auftauche (vgl. Weitin 2012, 219).

Es ist aber Tatsache, dass auch bei neueren Studien eine rege Diskussion um die Unterscheidung der Begriffe „Migrationsliteratur“ und „Migrantenliteratur“ weiter anhält, wie detailliert bei Klüh $(2009,45)$ dargestellt wird. In Anlehnung an Arens (2000) und Thore (2004) entscheidet sich Klüh für „Migrantenliteratur“, da sie den Begriff als Oberbegriff für „Minderheitenliteratur“ versteht, thematische Offenheit anstrebt und darunter nicht ausschließlich autobiographische Litera- 
tur assoziiert. Bei möglichen Einwänden ist jedoch auf ihre treffende Schlussfolgerung hinzuweisen, dass es in der entsprechenden Diskussion in Deutschland Begriffe im engeren Sinne und im offeneren Sinne, die also mehr als nur Biographie und Thema fokussieren, existieren und die Begriffswahl voneinander entschieden divergiert. ${ }^{3}$ Schließlich ist es sicherlich aber auch keine Lösung, nur auf die angebliche Unangemessenheit der Begriffe „Migrantenliteratur“ und „Migrationsliteratur“ hinzuweisen (vgl. Dörr 2006, 148). Die Begriffe voneinander zu unterscheiden würde stattdessen die Argumentation untermauern.

Ähnlichkeiten mit dem breit gefassten Migrationsbegriff weist der von Geiser geprägte Begriff der „Postmigration“ auf. Ähnlich wie der Migrationsbegriff fokussiert er die biographische Ebene, ohne sie auf die literarische Ebene im Sinne einer sprachlichen Einwanderung zu verlagern (vgl. Geiser 2015, 308). Das Präfix „post“ werde im Sinne Homi K. Bhabhas verwendet, nämlich als Vermittlung zwischen Vergangenem und Zukünftigem (vgl. Geiser 2015, 308). Die Pointe dieses Begriffs ist - was ihn hauptsächlich vom breit gefassten Begriff der „Migrationsliteratur“ unterscheidet -, dass er sich ausschließlich auf das Schreiben der nachkommenden Migrationsgeneration bezieht. Dabei ist m. E. bedenklich, ob solch eine Zäsur in den Themen und Schreibweisen tatsächlich besteht. Als Beispiel sei hier der Roman des Autors der zweiten Generation Alexandros Stefanidis Beim Griechen zu nennen, dessen thematischen Rahmen sehr wohl die zweite Generation bildet, diese aber auf den Lebenserfahrungen der Eltern und Großeltern (z. B. griechischer Bürgerkrieg) aufbaut und somit im wahrsten Sinne des Wortes gemeinsame deutsch-griechische Erinnerungsarbeit leistet. Bildet letztlich Migration in der Nachkriegszeit Deutschlands, die in der Folge der Anwerbung ausländischer Arbeitskräfte entstand, denn kein Kontinuum, wo sich diese translokalen Erfahrungen thematisch, sprachlich und ästhetisch quer durch die Generationen ziehen? In solch einem Kontext meint „Migrationsliteratur“ vornehmlich ein in Deutschland verankertes literarisches Phänomen, dessen gesellschaftlichen Referenzrahmen die Migration in Deutschland seit den sechziger Jahren bildet. Folglich stellt das literarische Schaffen der ersten Generation und der Folgegenerationen ein historisch-ästhetisches Kontinuum her, das die „deutsche Migrationsliteratur“ von anderen „Migrationsliteraturen“ in Europa unterscheidet. ${ }^{4}$

3 Ausführliche Beschreibungen der Unterscheidungen zwischen „Migrationsliteratur“ und „Migrantenliteratur“ vgl. Klüh (2009, 38-47). Eine detaillierte Darstellung würde den Rahmen des vorliegenden Beitrags sprengen.

$4 \mathrm{Ob}$ in einer supranationalen Betrachtungsweise von einer „europäischen Migrationsliteratur“ die Rede sein kann, die abgesehen vom Entstehungsort und -sprache gemeinsame migrantische Merkmale aufweist, ist eine komparatistisch brisante Frage, die an dieser Stelle dahingestellt bleiben muss. 
Geiser ist sicherlich zuzustimmen, dass die Biographie auch für Autoren der zweiten Generation eine wichtige Rolle spielt und der Fokus auf den Kontext der Migration gelegt werden sollte, zumal dieser die Lebenssituation der Autoren prägt (vgl. Geiser 304), wobei man im Bewusstsein behalten sollte, dass es sich um „eine Kombination von Merkmalen handelt, die möglicherweise das Schreiben beeinflussen, nicht jedoch per se ein Korpus bestimmen können.“ (Geiser 305 f.)

\section{Zur Hartnäckigkeit des Begriffs „Migrationsliteratur“}

Als Alternative zur „MigrantInnen- oder Migrationsliteratur“ wird eine „Literatur ohne festen Wohnsitz“ vorgeschlagen, die sich angeblich der Einordnung nach nationalphilologischen Kategorien entzieht und sprachlich-territoriale Grenzen überschreitet (vgl. Theilen 2005, 321). Theilen erkennt an, dass Migration ein Überbegriff ist, der die verschiedensten Formen der Bewegung im Raum zum Ausdruck bringt (vgl. Theilen 2005, 319), fragt sich aber bei aller Vielfalt des Migrationsphänomens, welches das Thema „der sogenannten MigrantInnenliteratur“ sein kann; solch eine Kategorie werde der Vielfalt der Texte nicht gerecht und ignoriere ästhetische Kategorien wie Struktur und Stil der Texte. Deshalb schlage sie eine Klassifikation nach strukturellen Merkmalen vor, eben jener „Literatur der Bewegung“, in der die Bewegung zum Strukturprinzip der Texte erhoben wird (Theilen 2005, 320). Bezeichnend ist, dass vornehmlich das Heranziehen der Biographie der Autoren kritisiert wird. Ähnlich behauptet Esselborn, dass man sich für den Begriff Migrationsliteratur aussprechen könne, wenn der Begriff konsequent für die vielfältigen und unterschiedlichen Wanderungserfahrungen der Autoren eingesetzt werde, was aber seiner Meinung nach den Begriff überfordere (vgl. Esselborn 2007, 261). Dem kann man natürlich widersprechen; Tatsache ist, dass alle Termini, die „Migranten- oder Migrationsliteratur“ ersetzen wollen, keine theoretischen Konzeptualisierungen zur Migration - was wird unter Migration im gesellschaftlichen und literarischen Kontext verstanden - liefern. Dies ist aber meines Erachtens essentiell.

Migration in einem dekonstruktivistischen Verständnis, wie sie Baltes-Löhr (1998, 86) verwendet, ist als Zirkulation zwischen Räumen zu verstehen, wobei Bipolaritäten (hier vs. dort, Heimat vs. Fremde) gesprengt werden. Die Definition von Migration, Migrantin und Migrant könne demzufolge aus ihrer binären Verfasstheit gelöst werden (vgl. Baltes-Löhr 2016, 23). Auch der Begriff der „Heimat“ kann zum Erinnerungsort und demzufolge zum Nicht-Ort vieler Migrantinnen 
und Migranten der Nachfolgegenerationen werden, da sich laut Barr ihre Identität sowohl in der Welt der Eltern als auch in der eigenen Außenwelt entfalte. Diese neue hybride Identität ,gehört somit zur Gesellschaft Deutschlands und letztlich zur modernen Form der Identitätskonstruktion, die wiederum zur subjektiven Definition der Heimat führt.“ (Barr 2016, 142)

Genau dieser breit und offen gefasste Begriff von „Migration“ liefert aber die Basis, um darauf aufbauend den Begriff der „Migrationsliteratur“ zu konzeptualisieren. Da in vielen literaturwissenschaftlichen Arbeiten nicht erklärt wird, was überhaupt unter Migration zu verstehen ist, schlagen sich die entsprechenden alternativen Definitionsversuche in der Opposition „Migrationsliteratur“ vs. anderer Vorschlag, meistens zum Nachteil der „Migrationsliteratur“, nieder. „Migrationsliteratur“ dient faktisch als Sprungbrett, um angeblich adäquatere Begriffe zu etablieren. Die gängige Vorgehensweise ist dabei: a) die entsprechende Forschungsliteratur zum Verständnis von „Migration“ kaum heranzuziehen und b) entsprechende Literatur zur „Migrationsliteratur“ selektiv zu Rate zu ziehen.

Chiara Cerri wirft z. B. die Frage auf, ob man Migrant lebenslang bleibe, und meint, dass der Begriff „Migrantenliteratur“ - auch sie verwendet die Begriffe „Migrantenliteratur“ und „Migrationsliteratur“ tautologisch - die „AutorInnen in einen Zustand der Daueremigration hinein“ zwinge (Cerri 2008, 429). Gegen den Begriff der „Migrationsliteratur“ spreche schlicht die Tatsache, dass der Begriff autobiographisch und authentisch sei, und daher entscheidet sie sich für „interkulturelle Literatur“, ohne jedoch Ansätze zum interkulturellen Potential der Migrationsliteratur heranzuziehen (Cerri 2008, 329). Auch verwundert diese Schlussfolgerung, da sie im von Blioumi verwendeten Begriff der „Migrationsliteratur“ interkulturelle Elemente ausgeschlossen sieht, obgleich sie einräumt, dass bei ihr die Koordinaten der Sprache, der Biographie und des Inhaltes als relationale Größen verstanden werden (vgl. Cerri 2006, 425). In ihrem späteren Artikel relativiert sie jedoch diese Position, erkennt an, dass Blioumi von einem nomadischen, also breiten Migrationsbegriff ausgeht, vereinnahmt aber Blioumis Ausführungen, wenn sie behauptet, dass gerade das Oszillieren zwischen den Räumen und Orten zum Konstitutionsmerkmal der „interkulturellen Literatur“ wird (vgl. Cerri 2011, 392). Schließlich beziehen Hofmann und Patrut $(2015,69)$ den Begriff der „interkulturellen Literatur“ entweder auf die Biographie der Autoren oder auf den Inhalt der Werke. ${ }^{5}$

5 Es muss jedoch betont werden, dass Hofmann und Patrut $(2015,69)$ in ihrer Argumentation den von ihnen bevorzugten Terminus der „interkulturellen Literatur“ nicht gegen die „Migrationsliteratur" ausspielen. 
Bei Wägenbaur $(1995,24)$ wird „interkulturelle Literatur“ explizit als Ersatz von „Gastarbeiter-, Ausländer- und Migrantenliteratur“" gebraucht, die undifferenziert verwendet werden, da angeblich bei dieser Literatur interkulturelle Elemente vermisst werden. Zweifelsohne aber ist an den Begriff ,interkulturelle Literatur“ die Erwartung geknüpft, in den literarischen Texten interkulturelle Elemente zu finden. ${ }^{6}$ Ähnlich plädiert zwar Dörr für die Verwendung des Begriffs „Migrantenliteratur", beugt aber spezifischen Rezeptionserwartungen mit der Warnung vor, dass diese Literatur nicht zwingend mit der Erwartung zu verknüpfen ist, darin eine migrantische Thematik zu finden (vgl. Dörr 2010, 86). Um nicht spezifische Rezeptionserwartungen auszulösen, warnt ähnlich Petra Günther vor der „Kolonisierung der Migrantenliteratur“, wenn letzte als Projektionsfläche für etwaige theoretische Ansätze verwendet werde. So sei bei der amerikanischen Germanistik spätestens seit Mitte der neunziger Jahre das Bemühen, diese Literatur an die cultural studies anzubinden, festzustellen, zumal sie ertragreiche Ergebnisse in Bezug auf die Bezugsfelder der cultural studies par excellence - Kultur, Identität, Hybridität - verspreche. Folglich werde die „deutschsprachige Migrantenliteratur als die einer nun endlich zu Wort kommenden Minderheit gedeutet.“ (Günther 2002, 153)

Die vorgeschlagenen Alternativen aber wirken sich gerade auf dieses literarische Phänomen verschleiernd aus, da positive Momente einer literarisch avancierten Migrationsliteratur, die eben mit dem Begriff transportiert werden, durch einen alternativen Vorschlag ohne die Semantik der Migration wegfallen, folglich „Migrationsliteratur“ wie einst „Gastarbeiterliteratur“ schlicht marginalisiert wird. Der Transfer des externen Begriffs „Migration“ auf literaturwissenschaftlicher Ebene bringt sehr wohl auch ästhetische Kategorien zum Ausdruck, wie z. B. das interkulturelle Potential der Migrationsliteratur. Nach Leskovec - sie verwendet den Begriff „Migrantenliteratur“ - handelt es sich hierbei um eine Literatur, die Fremdheit sowohl thematisch als auch sprachlich durch ästhetische Verfahren inszeniert (vgl. Leskovec 2011, 62 f.)

Eines der ersten Werke, das dieses literarische Phänomen als „interkulturelle Literatur" bezeichnet, ist das erste diesbezügliche Handbuch Carmine Chiellinos mit dem Titel Interkulturelle Literatur in Deutschland, wo für die Überwindung einer bipolaren, kultur-ethnischen Auslegung der Texte plädiert wird (vgl. Chiellino 2000, 394). Da seitdem das Label ,interkulturell“ inflationär verwendet wird, ohne sich unbedingt an Chiellino anzulehnen, ist hierbei anzumerken, dass für eine funktionale Begriffswahl zuvorderst der Begriff der „Interkulturalität“ (vgl.

6 Eingehende Beschäftigung mit Wägenbaur’s Begriff der interkulturellen Literatur vgl. Blioumi 2001, 117. 
Gutjahr 2010) und folglich die „literarische Interkulturalität“ (vgl. Schmitz 2009, $8)^{7}$, ähnlich wie bei der „Migration“ und der „Migrationsliteratur“, definiert werden sollten. Diejenigen Merkmale, die in den neuesten Debatten immer wieder als die Konsistenz dieser Literatur betrachtet werden - wie z. B. topographische Themen, interkulturelle Erfahrungen, Motive der Reise und des Nomadismus (vgl. Schmitz 2009), besonderer Umgang mit der Sprache (vgl. Federmair 2007, 418), stilistische und sprachliche Mischung (vgl. Joachimsthaler 2009, 34), positive Konnotierung der gebrochenen Sprache (vgl. Mein 2004, 203), Schreibweisen einer kulturellen Vielfalt (vgl. Schenk et al. X) im Sinne von beweglichen Poetiken (vgl. Federmair 2007, 416), die zur „migrantischen Qualität“ (Federmair 2012, 156) dieser Literatur führen -, machen schließlich das interkulturelle Potenzial der „Migrationsliteratur“ aus. Nach Leskovec $(2011,13)$ sei ein zusätzliches Kriterium zur Bestimmung des interkulturellen Potentials die Funktion der Literatur in interkulturellen Kommunikationssituationen sowie das ,Einüben“ von interkulturellen Kompetenzen, was sich aber gering auf die literarische Beschaffenheit der Texte bezieht. Mit Geiser bin ich schließlich an dieser Stelle einig, dass das Attribut ,interkulturell“ weder als Korpus- noch als Gattungsbegriff dienlich ist. Es bezeichnet ,entweder eine Schreibhaltung, bestimmte kommunikative Eigenschaften von Texten oder auch eine Rezeptionshaltung und methodische Lesart, [...] die sich ganz allgemein auf alle literarischen Produktionssituationen, die durch Kulturkontakte geprägt sind“ (Geiser 2015, 309), beziehen. Die Diskussion um ,interkulturelle Literatur“, die zur Substitution der „Migrationsliteratur“ vorgeschlagen wird, verkennt, dass auch „Migrationsliteratur“ heuristisch verstanden wird (vgl. Cerri 2006, 425). Ein breit verstandener Migrationsbegriff und in der Folge Migrationsliteraturbegriff fokussiert nicht nur inhaltliche Aspekte, sondern auch ästhetisch-interkulturelle Konfigurationen im Migrationskontext.

Trotz obiger Feststellung gebührt ein Wort Carmine Chiellinos zuletzt erschienener Studie $A B C$ für interkulturelle Leser (2016), die für komparatistische Herangehensweisen unentbehrlich ist, zumal sie sich nicht auf eine Nationalliteratur begrenzt, sondern den Forschungsgegenstand auf Literaturen in Westeuropa und Nordamerika ausweitet. Durch das Attribut ,interkulturell“, das sowohl den Produktions- als auch den Rezeptionsbedingungen gilt, werden zwar negative Konnotationen des Migrationsbegriffs umgangen, doch die Hauptkonstituenten der Migrationsliteraturen, nämlich das Zusammenspiel der Bewegung im Raum,

7 Wie schwierig das jedoch ist, zeigt die Kritik von Schmitz gegenüber Hofmann, demzufolge Hofmanns Begriff des Interkulturellen „gleichzeitig zu weit und zu eng gefasst“ sei (Schmitz 2009, 8). Vgl. die eingehenden Beschreibungen zur „interkulturellen Literaturwissenschaft“ bei Hofmann 2006. 
der Biographie und somit auch der Sprache, in den Vordergrund gerückt. Interpretatorischer Ausgangspunkt ist die wesentliche, aber in der Forschung wenig beachtete Konstitution eines „interkulturellen Gedächtnisses“, das insofern Sprachinnovation bedeutet, als dass die Sprache nicht nur Trägerin des kulturhistorischen Gedächtnisses (vgl. Chiellino 2016, 48) ist, sondern dass sie es vermag, die Geschichte verschiedener Herkunftsländer und -kulturen in die jeweilige Sprache der Literatur der Aufnahmeländer einzuflechten. Chiellino spricht von der „interkulturellen Literatur Europas“, weil durch diese Literaturen die zeitgenössische Geschichte der Herkunftsländer der Autoren Teil des Gedächtnisses der Sprachen, in denen die Werke geschrieben sind, werden (vgl. Chiellino 2016, 82). Statt Migrationsautoren verwendet Chiellino den Begriff „Sprachwechsler“ (Chiellino 2016, 205), was m. E. den Kern der Migrationsliteratur trifft.

Es ist jedoch nicht von der Hand zu weisen, dass aus komparatistischer Sicht ein Begriff wie „deutschsprachige Migrationsliteratur“ nicht ganz unproblematisch ist. Der Widerspruch besteht darin, dass einerseits das Phänomen territorial definiert wird, um den historisch-gesellschaftlichen Rahmenbedingungen und seinen literaturgeschichtlichen Existenzbedingungen (z. B. Kontinuum seit den sechziger Jahren) zu entsprechen, anderseits der breit aufgefasste Migrationsbegriff und folglich Migrationsliteraturbegriff Zirkulationen in diversen kulturellen Räumen, Transgressionen, Grenzüberschreitungen, Mehrsprachigkeit und patchwork-Identitäten meint. Der Widerspruch könnte dadurch gelöst werden, wenn komparatistische Studien im Sinne von „europäischen Migrationsliteraturen“ verstärkt Berücksichtigung fänden, da dadurch die Kontexte der Migration in Europa miteinander in Beziehung zu setzen wären. ${ }^{8}$

Schließlich widmet sich das zuletzt erschienene Sammelwerk von Rădulescu und Baltes-Löhr Pluralität als Existenzmuster (2016) der deutschsprachigen Migrationsliteratur, was die Tatsache bestätigt, dass sich der Terminus hartnäckig hält. Viele Beiträge unterstreichen die veränderte Rezeptionshaltung und betonen die hohe Akzeptanz, die heutzutage Autorinnen und Autoren mit Migrationshintergrund auf dem Literaturmarkt genießen (vgl. Rădulescu 2016, 177). Das „Etikett der Migrationsliteratur [sorgt] für Erfolge auf dem Buchmarkt“ (Krauze-Olejniczak 2016, 113). Ebenso für die Wissenschaft scheint sich die Praktikabilität des Begriffs bewährt zu haben, zumal allmählich Einsicht darin besteht, dass Migrationsliteratur sowohl auf der „biographischen Erfahrung der Einzelnen als auch auf

8 Hierzu siehe exemplarisch die Dissertation von Myriam Geiser (2015), die komparatistisch die deutsch-türkische und die frankomaghrebinische Literatur behandelt. Eine wahrhaftige Fundgrube ist die neu eröffnete Chiellino-Bibliothek. Eine Forschungsstelle für Literatur und Migration an der Europa-Universität Viadrina. 
der imaginativen Erkenntnis und der transkulturellen Kompetenz basieren kann“ (Krauze-Olejniczak 2016, 101).

Fazit: Begriffsbildungen sind sowohl mit den Produktions- als auch mit den Rezeptionsbedingungen verbunden. Zum einen rekurrieren Begriffe zu dieser Literatur auf die stetigen Veränderungen innerhalb der deutschen Realität (Gastarbeiter, Ausländer, Migrant), zum anderen beziehen sie sich auf die spezifischen Schwerpunkte der wissenschaftlichen Empfänger. Da die Labilität der Begriffszuweisungen die zentrale Problematik um dieses literarische Phänomen bestimmt, stimme ich mit der Meinung Federmairs überein, dass die „sprach-, kultur- und inhaltsbezogenen Sichtweisen“ analog zur Flexibilität der Metaebene zu sichten sind (Federmair 2012, 154) - und der jeweiligen gesellschaftlichen Realität, würde ich hinzufügen. Im Fall der Migrationsliteratur handelt es sich schließlich um einen rezeptionsstarken Terminus, der breit zu verstehen ist, um damit die historischen Entwicklungsetappen des literarischen Phänomens nicht zu verkennen und ebenso dessen ästhetische Dynamik, die vielfältig auf den Migrationsdiskurs reagiert, zu berücksichtigen. Unverkennbar also auch hier die These der Wechselwirkung zwischen Literatur und Realität, und dies sollte man vielleicht als Label dieser Literatur festhalten, im Bewusstsein jedoch, dass Wissenschaft ebenso unter Umständen Schubladendenken forciert - oder am besten entkräftet.

\section{Literaturverzeichnis}

Abel, Julia. „Positionslichter. Die neue Generation von Anthologien der Migrationsliteratur“. Text+Kritik XI (2006): 233-245.

Ackermann, Irmgard. „Zur Standortbestimmung der „Ausländerliteratur“. Literatur im interkulturellen Kontext. TUB-Dokumentationen, H. 20. Hg. Heidi Rösch. Berlin: Univ.-Bibliothek der Technischen Universität, 1993. 55-57.

Adelson, Leslie. „Migrantenliteratur oder deutsche Literatur? TORKANs Tufan: Brief an einen islamischen Bruder." Spätmoderne und Postmoderne. Beiträge zur deutschsprachigen Gegenwartsliteratur. Hg. Paul Michael Lützeler. Frankfurt/M.: Fischer, 1991. 67-81.

Amodeo, Immacolata. Die Heimat heißt Babylon. Zur Literatur ausländischer Autoren in der Bundesrepublik Deutschland. Opladen: Westdeutscher Verlag, 1996.

Arens, Hiltrud. ,Kulturelle Hybridität“ in der deutschen Minoritätenliteratur der achtziger Jahre. Tübingen: Stauffenburg, 2000.

Baltes-Löhr, Christel. „Frauen in der Migration: Dekonstruktivistische Analyse der Begriffe ,Identität' - ,Migration“ - ,Raum““. Perspektiven der Frauenforschung: ausgewählte Beiträge der 1. Fachtagung Frauen-, Gender-Forschung in Rheinland-Pfalz. Hg. Renate von Bardeleben und Patricia Plummer. Tübingen: Stauffenburg, 1998. 81-98.

Baltes-Löhr, Christel. „Die Figur des Kontinuums am Beispiel von Geschlecht und Migration. Ein Erklärungsansatz für Pluralitäten als Existenzmuster?“ Pluralität als Existenzmuster. 
Interdisziplinäre Perspektiven auf die deutschsprachige Migrationsliteratur. Hg. Raluca Rădulescu und Christel Baltes-Löhr. Bielefeld: transkript, 2016. 9-28.

Barr, Anja. „Die Heimat der Heimatlosen. Transkulturelle Identitäten in Özdamars Der Hof im Spiegel und Fatih Akins Gegen die Wand“. Pluralität als Existenzmuster. Interdisziplinäre Perspektiven auf die deutschsprachige Migrationsliteratur. Hg. Raluca Rădulescu und Christel Baltes-Löhr. Bielefeld: transkript, 2016. 139-152.

Blioumi, Aglaia: ,,Migrationsliteratur', ,interkulturelle Literatur' und ,Generationen von Schriftstellern'. Ein Problemaufriß über umstrittene Begriffe“. Weimarer Beiträge 46,4 (2000): 595-601.

Blioumi, Aglaia. Interkulturalität als Dynamik. Ein Beitrag zur deutsch-griechischen Migrationsliteratur seit den siebziger Jahren. Tübingen: Stauffenburg 2001.

Blioumi, Aglaia. Transkulturelle Metamorphosen. Deutschsprachige Migrationsliteratur im Ausland am Beispiel Griechenland. Monographie - Anthologie. Würzburg: Königshausen \& Neumann, 2006.

Cerri, Chiara. „Interkulturelle Literatur. Ein erneutes Plädoyer für eine dringende begriffliche Entscheidung“. Weimarer Beiträge 54.3 (2008): 424-436.

Cerri, Chiara. „Mut zur interkulturellen Literatur im DaF-Unterricht“. Info-DaF38.4 (2011): 391-413.

Chiellino, Carmine. „Interkulturalität und Literaturwissenschaft“. Interkulturelle Literatur in Deutschland. Ein Handbuch. Hg. Chiellino Carmine. Stuttgart und Weimar: Metzler, 2000. 387-398.

Chiellino, Carmine: Interkulturell Literatur in deutscher Sprache. Das große ABC für interkulturelle Leser. Bern, Berlin u. a.: Lang, 2016.

Dörr, Volker C. „,Third Space“ vs. Diaspora. Topologien transkultureller Literatur“. Von der nationalen zur internationalen Literatur: transkulturelle deutschsprachige Literatur und Kultur im Zeitalter globaler Migration. Hg. Walter Schmitz. Amsterdam: Rodopi, 2009. 59-76.

Dörr, Volker C. „,Gastarbeiter‘ vs. ,Kanakstas‘: Migranten-Biographien zwischen Alterität, Hybridität und Transkulturalität". AutoBioFiktion. Konstruierte Identitäten in Kunst, Literatur und Philosophie. Hg. Christian Moser und Jürgen Nelles Bielefeld: Aisthesis, 2006. 145-165.

Dörr, Volker C. „Multi-, Inter-, Trans- und Hyper-Kulturalität und (deutsch-türkische) ,Migrantenliteratur'“. Zwischen Provokation und Usurpation. Interkulturalität als (un)vollendetes Projekt der Literatur- und Sprachwissenschaften. Hg. Dieter Heimböckel, Irmgard HonnefBecker und Georg Mein. München: Fink, 2010. 71-86.

Esselborn, Karl. „Deutschsprachige Literatur von Autoren nichtdeutscher Muttersprache und der Adelbert-von-Chamisso-Preis“. Jahrbuch Deutsch als Fremdsprache 21 (1995): 411-427.

Esselborn, Karl. „,Übersetzungen aus der Sprache, die es nicht gibt.“ Interkulturalität, Globalisierung und Postmoderne in den Texten Yoko Tawadas“. arcadia 42.2 (2007): 240-262.

Federmair, Leopold. „Sprachspiel und Interkulturalität“. Weimarer Beiträge 53.3 (2007): 412-426.

Federmair, Leopold. „Der neue Diamant: Verfremdungseffekte bei E. S. Özdamar“. arcadia 47.1 (2012): 151-172.

Geiser, Myriam. Der Ort transkultureller Literatur in Deutschland und in Frankreich. Deutschtürkische und frankomaghrebinische Literatur der Postmigration. Würzburg: Königshausen \&Neumann, 2015. 
Günther, Petra. „Die Kolonisierung der Migrantenliteratur“. Räume der Hybridität. Postkoloniale Konzepte in Theorie und Literatur. Hg. Christof Hamann und Cornelia Sieber. Hildesheim, Zürich und New York: Olms, 2002, 151-160.

Gutjahr, Ortrud. „Interkulturalität als Forschungsparadigma der Literaturwissenschaft. Von den Theoriedebatten zur Analyse kultureller Tiefensemantiken“. Zwischen Provokation und Usurpation. Interkulturalität als (un)vollendetes Projekt der Literatur- und Sprachwissenschaften. Hg. Dieter Heimböckel, Irmgard Honnef-Becker und Georg Mein. München: Fink, 2010. 17-40.

Hamm, Horst: Fremdgegangen - freigeschrieben. Eine Einführung in die deutschsprachige Gastarbeiterliteratur. Würzburg: Königshausen \& Neumann, 1988.

Hinnenkamp, Volker: „Gastarbeiterlinguistik und die Ethnisierung der Gastarbeiter“. Ethnizität: Wissenschaft und Minderheiten. Hg. Eckhard J. Dittrich und Frank-Olaf Radtke. Opladen: Westdeutscher Verlag, 1990. 277-297.

Hofmann, Michael. Interkulturelle Literaturwissenschaft. Paderborn: Fink/UTB, 2006.

Hofmann, Michael, und Iulia-Karin Patrut (Hg.). Einführung in die interkulturelle Literatur. Darmstadt: WBG, 2015.

Joachimsthaler, Jürgen. „,Undeutsche Bücher': Zur Geschichte interkultureller Literatur in Deutschland“. Von der nationalen zur internationalen Literatur: transkulturelle deutschsprachige Literatur und Kultur im Zeitalter globaler Migration. Hg. Walter Schmitz. Amsterdam: Rodopi, 2009.17-39.

Kliems, Alfrun. „Migration - Exil - Postkolonialismus? Reflexionen zu Kanonisierung und Kategorisierung von Literatur.“ Migrationsliteratur. Schreibweisen einer interkulturellen Moderne. Hg. Klaus Schenk, Almut Todorow und Milan Tvrdik. Tübingen und Basel: Francke, 2004. 287-300.

Klüh, Ekaterina. Interkulturelle Identitäten im Spiegel der Migrantenliteratur. Kulturelle Metamorphosen bei Ilija Trojanow und Rumjana Zacharieva. Würzburg: Königshausen und Neumann, 2009.

Krauze-Olejniczak, Alicia. „Migrationsroman, der gar keiner sei: Malin Schwerdtfegers Café Saratoga“. Pluralität als Existenzmuster. Interdisziplinäre Perspektiven auf die deutschsprachige Migrationsliteratur. Hg. Raluca Rădulescu und Christel Baltes-Löhr. Bielefeld: transkript, 2016. 97-116.

Leskovec, Andrea. Einführung in die interkulturelle Literaturwissenschaft. Darmstadt: WBG, 2011.

Lübcke, Alexandra. „Enträumlichungen und Erinnerungstopographien: Transnationale deutschsprachige Literaturen als historiographisches Erzählen." Von der nationalen zur internationalen Literatur: transkulturelle deutschsprachige Literatur und Kultur im Zeitalter globaler Migration. Hg. Walter Schmitz. Amsterdam: Rodopi, 2009. 77-98.

Moser, Natalie. „Deutschsprachige Migrationsliteratur in der Schweiz? Zur Prosa von Cătălin Dorian Florescu“. Pluralität als Existenzmuster. Interdisziplinäre Perspektiven auf die deutschsprachige Migrationsliteratur. Hg. Raluca Rădulescu und Christel Baltes-Löhr. Bielefeld: transkript, 2016.173-190.

Weinrich, Harald. „Gastarbeiterliteratur in der Bundesrepublik Deutschland“. LiLi 14 (1984): 14-22.

Mein, Georg. „Die Migration entlässt ihre Kinder. Sprachliche Entgrenzungen als Identitätskonzept.“ Deutschsprachige Gegenwartsliteratur seit 1989. Zwischenbilanzen Analysen - Vermittlungsperspektiven. Hg. Clemens Kammler und Torsten Pflugmacher. Heidelberg: Sychron, 2004. 201-217. 
Rădulescu, Raluca, und Christel Baltes-Löhr (Hg.). Pluralität als Existenzmuster. Interdisziplinäre Perspektiven auf die deutschsprachige Migrationsliteratur. Bielefeld: transkript, 2016.

Rădulescu, Raluca. „Die Lyrik José F. A. Olivers. Versuch einer ,modernen“ interkulturellen Hermeneutik“. Pluralität als Existenzmuster. Interdisziplinäre Perspektiven auf die deutschsprachige Migrationsliteratur. Hg. Raluca Rădulescu und Christel Baltes-Löhr. Bielefeld: transkript, 2016. 63-78.

Rösch, Heidi. Migrationsliteratur im interkulturellen Kontext: Eine didaktische Studie zur Literatur von Aras Ören, Aysel Özakin, Franco Biondi und Rafik Schami. Frankfurt a. M.: IKO, 1992.

Schenk, Klaus, und Almut Todorow, und Milan Tvrdik (Hg.). Migrationsliteratur. Schreibweisen einer interkulturellen Moderne. Tübingen und Basel: Francke, 2004.

Schierloh, Heimke. Das alles für ein Stück Brot. Migrantenliteratur als Objektivierung des „Gastarbeiterdaseins“ mit einer Textsammlung. Frankfurt/M.: Peter Lang, 1984.

Schmitz, Helmut. „Einleitung“. Von der nationalen zur internationalen Literatur: transkulturelle deutschsprachige Literatur und Kultur im Zeitalter globaler Migration Hg. Walter Schmitz. Amsterdam: Rodopi, 2009. 7-15.

Theilen, Ines. „Von der nationalen zur globalen Literatur. Eine Lese-Bewegung durch die Romane Die Brücke vom goldenen Horn von Emine Sevgi Özdamar und Cafe Nostalgia von Zoe Valdes“. arcadia 40/321.2 (2005): 318-337.

Thore, Petra. „wer bist du hier in dieser stadt, in diesem land, in dieser neuen welt“. Die Identitätsbalance in der Fremde in ausgewählten Werken der deutschsprachigen Migrantenliteratur. Uppsala: Uppsala universitet, 2004.

Wägenbaur, Thomas. „Kulturelle Identität oder Hybridität. Aysel Özakins Die blaue Maske und das Projekt interkultureller Dynamik“. LiLi 97 (1995): 22-47.

Weigel, Sigrid. „Literatur der Fremde - Literatur in der Fremde“. Gegenwartsliteratur seit 1968, Bd. 12. Hg. Sigrid Weigel und Klaus Briegleb. München: Hanser 1992. 182-229.

Weitin, Thomas. „Exil und Migration. Minoritäres Schreiben auf Deutsch im 20. Jahrhundert von Kafka zu Zaimoglu“. Weimarer Beiträge, 2.58 (2012): 195-224.

Zaimoglou, Feridun. „Migrationsliteratur ist ein toter Kadaver“. Text+Kritik XI (2006): 159-166.

Aglaia Blioumi, Assistant Prof. Dr., Promotion an der FU Berlin über deutschgriechische Migrationsliteratur. Assistenzprofessor in Germanistik an der Nationalen und Kapodistrischen Universität Athen. Forschungsschwerpunkte: Migrationsliteratur, kulturwissenschaftliche Germanistik, interkulturelle Literaturwissenschaft, Reiseliteratur, Literaturdidaktik. Bis 2017 Stiftungsratsvorsitzende der Adamas-Stiftung Götz Hübner. 